\title{
Is there merit in treating gestational diabetes mellitus?
}

Crowther CA, Hiller JE, Moss JR, McPhee AJ, Jeffries WS, Robinson JS; Australian Carbohydrate Intolerance Study in Pregnant Women (ACHOIS) Trial Group. Effect of treatment of gestational diabetes mellitus on pregnancy outcomes. N Engl J Med 2005;352(24): 2477-86.

Background: Optimal management of women with gestational diabetes remains controversial and contested. Although gestational diabetes is known to be a risk factor for macrosomia (birth weight $\geq 4 \mathrm{~kg}$ ), birth trauma and cesarean delivery, the few randomized clinical trials that exist have shown few or no benefits from treatment.

Design: Pregnant women between 16 and 30 weeks of gestation at 18 collaborating centres were screened for insulin intolerance. Those having 1 or more risk factors for gestational diabetes or who had results from a 50 -g oral glucose challenge test (defined as $\geq 7.8 \mathrm{mmol} / \mathrm{L}$ at $1 \mathrm{~h}$ ) underwent a 75-g oral glucose tolerance test at 24-34 weeks' gestation. Healthy women with a fasting plasma-glucose measurement below $7.8 \mathrm{mmol} / \mathrm{L}$ but a 2 -hour result of $7.8-11 \mathrm{mmol} / \mathrm{L}$ were eligible for the study. Women with more severe glucose intolerance were excluded.

After randomization, women in the intervention group $(n=$ 490) were given dietary counselling, were taught how to selfmonitor glucose and received insulin therapy as needed to maintain fasting and premeal plasma glucose concentrations below $5.5 \mathrm{mmol} / \mathrm{L}$. The women in the control group $(n=510)$ received the care routinely given when screening for gestational diabetes is unavailable. These women (or their physicians) $\sim$ were told that they did not have gestational diabetes.

Primary outcomes among infants were serious perinatal complications (death, shoulder dystocia, bone fracture or nerve palsy), admission to the neonatal nursery and jaundice requiring pho- totherapy. The primary maternal outcomes of interest were induced labour, cesarean section and mental health scores (measured with the 36-item ShortForm General Health Survey, the short Spielberger StateTrait Anxiety Inventory and the Edinburgh Postnatal Depression Scale). All statistical analyses were based on intention to treat.

Results: Participants in the study were largely white (average age $30 \mathrm{yr}$ ) and primiparous.

Serious perinatal complications were significantly less frequent in the intervention group than in the routine-care group ( $1 \%$ v. $4 \%$; risk ratio [RR] 0.32 , $95 \%$ confidence interval [CI] $0.14-0.73)$. No infants died in the intervention group, but there were 5 deaths in the control group ( $p=0.06,2$-tailed). However, more babies were admitted to the neonatal nursery from the intervention group than the control group $(71 \% \mathrm{v}$. $61 \%$; RR 1.15 , 95\% CI 1.05 1.26). The proportion of infants with jaundice in both groups was $9 \%$. Labour was induced more frequently in the intervention group (39\% v. 29\%, RR 1.31 , 95\% CI 1.10-1.56), but rates of cesarean delivery differed little.

Infants born to the intervention group were less likely to be large for their gestational age (13\% v. $22 \%$; adjusted treatment effect 0.62 , 95\% CI $0.47-$ 0.81). Although women in the intervention group had fewer antenatal visits after enrolment, they made more visits postpartum and were also more likely to visit a dietitian and diabetes educator than those in the control group.

Anxiety levels reported by women who returned a second health questionnaire 3 months after giving birth were similar between the groups, but those in the intervention group were less likely to experience depression ( $8 \%$ v. $17 \%$ of controls).

Commentary: Whether gestational diabetes should be treated has generally been left to the discretion of the primary caregiver. In light of the results of this study, however, treatment may reduce serious perinatal outcomes. Three caveats may apply. First, because the physicians attending to the intervention group knew that their patients had gestational diabetes, they may have intervened more readily, thus biasing the results toward shorter gestations and fewer perinatal complications than among controls. Second, the small percentage of infants with macrosomia who have shoulder dystocia do not generally have any permanent injury. Third, the predominantly white, educated population may be unrepresentative.

Practice implications: In the United States, gestational diabetes mellitus is diagnosed in $3 \%-7 \%$ of pregnant women. If their quality of life and the health of their infants can be improved with treatment of the condition, then perhaps gestational diabetes should enter into the screening arena. Women with mild gestational diabetes can then receive dietary advice and insulin to maintain low fasting and premeal plasma glucose concentrations.

Ana M. Cartagena

Class of 2008

University of Manitoba

Winnipeg, Man.

\section{Reference}

1. Greene MF, Solomon CG. Gestational diabetes mellitus: time to treat [editorial]. N Engl 7 Med 2005;352 (24):2544-6 